\title{
Low-velocity impact response of a pre-stressed isotropic Uflyand-Mindlin plate
}

\author{
Yury Rossikhin ${ }^{1, \star}$, Marina Shitikova ${ }^{1, \star \star}$, and Phan Thanh Trung ${ }^{1,2}$ \\ ${ }^{1}$ Voronezh State Technical University, Research Center on Dynamics of Solids and Structures, Voronezh 394006, Russia \\ ${ }^{2}$ HCMC University of Technology and Education, Vietnam
}

\begin{abstract}
The low-velocity impact response of a precompressed circular isotropic elastic plate is investigated in the case when the dynamic behavior of the plate is described by equations taking the rotary inertia and transverse shear deformations into account. Contact interaction between the rigid impactor and the target is modeled by a generalized Hertz contact force, since it is assumed that the viscoelastic features of the plate represent themselves only in the place of contact and are governed by the standard linear solid model with fractional derivatives due to the fact that during the impact process decrosslinking occurs within the domain of the contact of the plate with the sphere, resulting in more free displacements of molecules with respect to each other, and finally in the decrease of the plate material viscosity in the contact zone.
\end{abstract}

\section{Introduction}

An impact response analysis requires a good estimate of contact force throughout the impact duration. Low velocity impact problems, which also took the local indentation into account, have been solved by many authors. Reference to the state-of-the-art reveiws $[1,4]$ shows that in most studies it was assumed that the impacted structure was free of any initial stresses. But this does not adequately reflect the real multidirectional complex loading states that the materials experience during their service life. The detailed reveiw of papers wherein prestressing of targets was taken into account during the solution of dynamic problems dealing with impact interaction could be found in [5]. But there is practically no investigations on viscoelasticity influence during the impact interaction.

In the present paper, we generalize the approaches suggested in [5] for study of the impact response of a prestressed elastic circular plate and in [6] for investigating the impact response of a plate, viscoelastic features of which are induced within the contact domain. But distinct to [6], in the problem under consideration the generalized Hertz contact law is utilized by considering timedependent operators describing rigidity and Poisson's ratio of plate's material.

\section{Problem formulation and methods of solution}

Let us consider the problem of the impact of rigid sphere upon a pre-stressed circular isotropic Uflyand-Mindlin plate which is presumed to be of infinite extent in order

\footnotetext{
${ }^{\star}$ e-mail: yar@vgasu.vrn.ru

$\star \star$ e-mail: shitikova@vmail.ru
}

to ignore the waves reflected from its edges. The plate out of the contact zone is considered to be elastic, while within the contact domain its microstructure changes and it gains viscoelastic properties.

\subsection{Dynamic response of a circular elastic plate}

The equations of motion of a pre-stressed circular isotropic elastic Uflyand-Mindlin plate could be written in the polar coordinate system with the origin at the center of the plate in the following form [5]:

$$
\begin{gathered}
D\left(\frac{\partial^{2} \varphi}{\partial r^{2}}+\frac{1}{r} \frac{\partial \varphi}{\partial r}\right)-D \frac{\varphi}{r^{2}}+K \mu h\left(\frac{\partial w}{\partial r}-\varphi\right)=\rho \frac{h^{3}}{12} \ddot{\varphi}, \\
K \mu h\left(\frac{\partial^{2} w}{\partial r^{2}}-\frac{\partial \varphi}{\partial r}+\frac{1}{r} \frac{\partial w}{\partial r}-\frac{\varphi}{r}\right)=\rho h \ddot{w}+N\left(\frac{\partial^{2} w}{\partial r^{2}}+\frac{1}{r} \frac{\partial w}{\partial r}\right),
\end{gathered}
$$

where $w$ is the plate deflection, $\varphi$ is the angle of inclination of the normal to plate's middle surface, $r$ is the polar radius, $h$ is the plate thickness, $\rho$ is its density, $K=5 / 6$ is the shear coefficient, $N$ is the constant compression force acting in the radial direction, $D=E h^{3} / 12\left(1-v^{2}\right)$ is the bending rigidity, $E, \mu$, and $v$ are the longitudinal modulus, shear modulus, and Poisson's ratio, respectively, and an overdot denotes a derivative with respect to time $t$.

It is assumed that as a result of impact upon the plate, two transient waves (the surfaces of strong discontinuity) arise in it at the point of impact, which further propagate with velocities $G_{\alpha}(\alpha=1,2)$ along the plate in the form of diverging cylindrical surfaces-strips. Let us interpret the surface of strong discontinuity as a layer of small thickness $\delta$, within which the desired value $Z$ changes monotonically and continuously from the magnitude $Z^{+}$to the magnitude 
$Z^{-}$. Suppose that the ahead and back fronts of the shock layer arrive at a certain point with the fixed radius at the time instants $t$ and $t+\Delta t$, respectively, where $\Delta t$ is small. Inside the shock layer the following relationship

$$
\partial Z / \partial r \approx-G^{-1} \partial Z / \partial t
$$

is fulfilled.

A certain desired function $Z$ behind the fronts of wave surfaces $\Sigma^{(1)}$ and $\Sigma^{(2)}$ could be represented in terms of the ray series [7]

$$
\begin{aligned}
Z(r, t) & =\left.\sum_{\alpha=1}^{2} \sum_{k=0}^{\infty} \frac{1}{k !}[Z,(k)]^{(\alpha)}\right|_{t=\frac{r}{G_{\alpha}}}\left(t-\frac{r}{G_{\alpha}}\right)^{k} \\
& \times H\left(t-\frac{r}{G_{\alpha}}\right),
\end{aligned}
$$

where $\left[Z,{ }_{(k)}\right]^{(\alpha)}=\left[\partial^{k} Z / \partial t^{k}\right]^{(\alpha)}=Z_{,(k)}^{+(\alpha)}-Z,_{(k)}^{-(\alpha)}$ are discontinuities in the $k$ th-order time-derivatives of the desired function $Z$ on the waves surfaces $\Sigma_{\alpha}$, i.e., at $t=r / G_{\alpha}$, the upper indices " + "and " - "denote that the values are calculated immediately ahead of and behind the wave fronts, respectively, and $H\left(t-r / G_{\alpha}\right)$ is the unit Heaviside function.

Since the impact process is of short duration, then it is possible to restrict only by zero-order terms, i.e.,

$$
Z(r, t)=\left.\sum_{\alpha=1}^{2}[Z]^{(\alpha)}\right|_{t=\frac{r}{G_{\alpha}}} H\left(t-\frac{r}{G_{\alpha}}\right) .
$$

Writing Eqs. (1) and (2) within the shock layer with due account for (3), then integrating the obtained relationships over $t$ from $t$ to $t+\Delta t$ and tending $\Delta t \rightarrow 0$ yield

$$
\begin{gathered}
\rho G_{1}^{2}=\frac{E}{1-v^{2}}, \\
\rho G_{2}^{2}=K \mu-N / h .
\end{gathered}
$$

For further treatment we need to determine the transverse force $Q_{r}$. For this purpose we would split Eq. (2) in two equations

$$
\begin{aligned}
\frac{\partial Q_{r}}{\partial r}+\frac{1}{r} Q_{r} & =\rho h \ddot{w}+N\left(\frac{\partial^{2} w}{\partial r^{2}}+\frac{1}{r} \frac{\partial w}{\partial r}\right), \\
Q_{r} & =K \mu h\left(\frac{\partial w}{\partial r}-\varphi\right) .
\end{aligned}
$$

Differentiating (8) and (9) one time with respect to time, applying the condition of compatibility (3) with due account for (7), we obtain

$$
Q_{r}=-K \mu h G_{2}^{-1} W,
$$

where $W=\dot{w}$.

\subsection{Equations of motion of the contact domain and the impactor}

At $t>0$ the displacement of the sphere's center could be represented in the form

$$
z=w+\alpha,
$$

where $\alpha$ is the impactor's indentation due to the local bearing of target material within the contact domain.

Then the equation of motion of the part of the plate being in contact with the sphere and the equation of the sphere have the form

$$
\begin{aligned}
2 \pi a(t) Q_{r}+P(t)= & \rho h \pi a^{2}(t) \ddot{w}+2 \pi a(t) N \frac{\partial w}{\partial r}, \\
& m \ddot{z}=-P(t),
\end{aligned}
$$

where $a(t)$ is the radius of the contact domain, and $P(t)$ is the contact force.

Equations (12) and (13) could be solved with due account for formula (10), as well as considering the following initial conditions:

$$
\left.z\right|_{t=0}=0,\left.\quad \dot{z}\right|_{t=0}=V_{0},\left.\quad w\right|_{t=0}=0,\left.\quad \dot{w}\right|_{t=0}=0 .
$$

It is assumed that the viscoelastic features of the plate represent themselves only in the place of contact and are governed by the standard linear solid model with fractional derivatives. The matter is fact that during the impact process, decrosslinking occurs within the domain of the contact of the plate with the sphere, resulting in more free displacements of molecules with respect to each other, and finally in the decrease of the plate material viscosity in the contact zone. This circumstance allows one to describe the behaviour of the plate material within the contact domain by the standard linear solid model involving fractional derivatives, since variation in the fractional parameter (the order of the fractional derivative) enables one to control the viscosity of the plate material.

In this case, the generalized contact Hertz theory could be used to define the contact force

$$
P(t)=\frac{4 \sqrt{R}}{3} \widetilde{k} \alpha^{3 / 2}
$$

and the time-dependent radius of the contact domain is defined as

$$
a(t)=\sqrt{\alpha(t) R},
$$

where $R$ is the radius of the impactor, $\widetilde{k}$ is the timedependent operator

$$
\widetilde{k}=\frac{\widetilde{E}}{1-\widetilde{v}^{2}}
$$

dependent on the viscoelastic operators $\widetilde{E}$ and $\widetilde{v}$ which are valid within the contact domain and based on the fractional derivative standard linear solid model.

Thus, the operator corresponding to the Young's modulus has the form [8]

$$
\widetilde{E}_{1}=E_{\infty}\left[1-v_{\varepsilon} \ni_{\gamma}^{*}\left(\tau_{\varepsilon}^{\gamma}\right)\right] \quad(0 \leq \gamma \leq 1)
$$

where $E_{\infty}$ and $E_{0}$ are the non-relaxed (instantaneous modulus of elasticity, or the glassy modulus) and relaxed elastic (prolonged modulus of elasticity, or the rubbery modulus) moduli which are connected with the relaxation time $\tau_{\varepsilon}$ and retardation time $\tau_{\sigma}$ by the following relationship:

$$
\left(\frac{\tau_{\varepsilon}}{\tau_{\sigma}}\right)^{\gamma}=\frac{E_{0}}{E_{\infty}}
$$




$$
\begin{gathered}
v_{\varepsilon}=\frac{E_{\infty}-E_{0}}{E_{\infty}}=\frac{\Delta E}{E_{\infty}}, \\
\ni_{\gamma}^{*}\left(\tau_{i}^{\gamma}\right) Z(t)=\int_{0}^{t} \ni_{\gamma}\left(\frac{t-t^{\prime}}{\tau_{i}}\right) Z\left(t^{\prime}\right) d t^{\prime} \quad(i=\varepsilon, \sigma), \\
\ni_{\gamma}\left(\frac{t}{\tau_{i}}\right)=\frac{t^{\gamma-1}}{\tau_{i}^{\gamma}} \sum_{n=0}^{\infty} \frac{(-1)^{n}\left(t / \tau_{i}\right)^{\gamma n}}{\Gamma[\gamma(n+1)]},
\end{gathered}
$$

$\Gamma[\gamma(n+1)]$ is the Gamma-function, $\ni_{\gamma}\left(t / \tau_{i}\right)$ is Rabotnov's fractional exponential function [9] which at $\gamma=1$ goes over into the ordinary exponent, and operator $\ni_{\gamma}\left(\tau_{i}\right)$ transforms into operator $\ni_{1}^{*}\left(\tau_{i}\right)$. When $\gamma \rightarrow 0$, the function $\ni_{\gamma}\left(t / \tau_{i}\right)$ tends to the Dirac delta-function $\delta(t)$.

As numerous experiments with volume stresses and strains show, for the majority of materials the operator of volume extension-compression $\widetilde{K}$ is a constant value, that is

$$
\widetilde{K}=K_{\infty},
$$

where $K_{\infty}$ is a certain constant.

Now we could calculate the Poisson's operator $\widetilde{v}$ according to formula (23), which could be rewritten in the form

$$
\frac{\widetilde{E}}{1-2 \widetilde{v}}=\frac{E_{\infty}}{1-2 v_{\infty}}=3 K_{\infty},
$$

where $v_{\infty}$ is the non-relaxed magnitude of the Poisson's ratio.

Considering (18), from formula (24) we have

$$
\widetilde{v}=v_{\infty}+\frac{1}{2}\left(1-2 v_{\infty}\right) v_{\varepsilon} \ni_{\gamma}^{*}\left(\tau_{\varepsilon}^{\gamma}\right)
$$

For further treatment we should know the following operators:

$$
\begin{aligned}
& \frac{1}{1+\widetilde{v}}=\frac{1}{1+v_{\infty}+\frac{1}{2}\left(1-2 v_{\infty}\right) v_{\varepsilon} \ni_{\gamma}^{*}\left(\tau_{\varepsilon}^{\gamma}\right)}, \\
& \frac{1}{1-\widetilde{v}}=\frac{1}{1-v_{\infty}-\frac{1}{2}\left(1-2 v_{\infty}\right) v_{\varepsilon} \ni_{\gamma}^{*}\left(\tau_{\varepsilon}^{\gamma}\right)} .
\end{aligned}
$$

In order to calculate the operators in the right-hand side of (26) and (27), we assume that they have the following form:

$$
\begin{aligned}
& \frac{1}{1+\widetilde{v}}=\frac{1}{1+v_{\infty}}\left[1-B \ni_{\gamma}^{*}\left(t_{1}^{\gamma}\right)\right], \\
& \frac{1}{1-\widetilde{v}}=\frac{1}{1-v_{\infty}}\left[1+D \ni_{\gamma}^{*}\left(t_{2}^{\gamma}\right)\right],
\end{aligned}
$$

where $B, t_{1}$ and $D, t_{2}$ are yet unknown constants.

Equating the right sides of relationships (26), (28) and (27), (29), reducing the obtained expressions to the common denominator with due account for formula [8]

$$
\ni_{\gamma}^{*}\left(\tau_{\varepsilon}^{\gamma}\right) \ni_{\gamma}^{*}\left(\tau_{\sigma}^{\gamma}\right)=\frac{\tau_{\varepsilon}^{\gamma} \ni_{\gamma}^{*}\left(\tau_{\varepsilon}^{\gamma}\right)-\tau_{\sigma}^{\gamma} \ni_{\gamma}^{*}\left(\tau_{\sigma}^{\gamma}\right)}{\tau_{\varepsilon}^{\gamma}-\tau_{\sigma}^{\gamma}}
$$

yield

$$
\begin{aligned}
\frac{1}{2} \frac{\left(1-2 v_{\infty}\right) v_{\varepsilon}}{1+v_{\infty}} & {\left[1-B \frac{\tau_{\varepsilon}^{\gamma}}{\tau_{\varepsilon}^{\gamma}-t_{1}^{\gamma}}\right] \ni_{\gamma}^{*}\left(\tau_{\varepsilon}^{\gamma}\right) } \\
& -B\left[1-\frac{1}{2} \frac{\left(1-2 v_{\infty}\right) v_{\varepsilon}}{\left(1+v_{\infty}\right)}\right. \\
& \left.\times \frac{t_{1}^{\gamma}}{\left(\tau_{\varepsilon}^{\gamma}-t_{1}^{\gamma}\right)}\right] \ni_{\gamma}^{*}\left(t_{1}^{\gamma}\right)=0,
\end{aligned}
$$

$$
\begin{aligned}
-\frac{1}{2} \frac{\left(1-2 v_{\infty}\right) v_{\varepsilon}}{1-v_{\infty}} & {\left[1+D \frac{\tau_{\varepsilon}^{\gamma}}{\tau_{\varepsilon}^{\gamma}-t_{2}^{\gamma}}\right] \ni_{\gamma}^{*}\left(\tau_{\varepsilon}^{\gamma}\right) } \\
+ & D\left[1+\frac{1}{2} \frac{\left(1-2 v_{\infty}\right) v_{\varepsilon}}{\left(1-v_{\infty}\right)}\right. \\
& \left.\times \frac{t_{2}^{\gamma}}{\left(\tau_{\varepsilon}^{\gamma}-t_{2}^{\gamma}\right)}\right] \ni_{\gamma}^{*}\left(t_{2}^{\gamma}\right)=0 .
\end{aligned}
$$

Vanishing to zero the expressions in square brackets in (31) and (32), we determine unknown constants

$$
\begin{gathered}
B=\frac{\left(1-2 v_{\infty}\right) v_{\varepsilon}}{2\left(1+v_{\infty}\right)+\left(1-2 v_{\infty}\right) v_{\varepsilon}}=\frac{\tau_{\varepsilon}^{\gamma}-t_{1}^{\gamma}}{\tau_{\varepsilon}^{\gamma}}, \\
t_{1}^{-\gamma}=\tau_{\varepsilon}^{-\gamma}\left[1+\frac{\left(1-2 v_{\infty}\right) v_{\varepsilon}}{2\left(1+v_{\infty}\right)}\right], \quad t_{1}^{\gamma}=\frac{\tau_{\varepsilon}^{\gamma}}{A}, \\
D=\frac{\left(1-2 v_{\infty}\right) v_{\varepsilon}}{2\left(1-v_{\infty}\right)-\left(1-2 \sigma_{\infty}\right) v_{\varepsilon}}=\frac{\tau_{\varepsilon}^{\gamma}-t_{2}^{\gamma}}{\tau_{\varepsilon}^{\gamma}}, \\
t_{2}^{-\gamma}=\tau_{\varepsilon}^{-\gamma}\left[1-\frac{\left(1-2 v_{\infty}\right) v_{\varepsilon}}{2\left(1-v_{\infty}\right)}\right], \quad t_{2}^{\gamma}=\frac{\tau_{\varepsilon}^{\gamma}}{C} \\
A=\frac{2\left(1+v_{\infty}\right)+\left(1-2 v_{\infty}\right) v_{\varepsilon}}{2\left(1+v_{\infty}\right)}, \\
C=\frac{2\left(1-v_{\infty}\right)-\left(1-2 v_{\infty}\right) v_{\varepsilon}}{2\left(1-v_{\infty}\right)} .
\end{gathered}
$$

Now we could calculate the operator

$$
\widetilde{k}=\frac{\widetilde{E}}{1-\widetilde{v}^{2}}=\frac{\widetilde{E}}{2}\left(\frac{1}{1+\widetilde{v}}+\frac{1}{1-\widetilde{v}}\right) .
$$

For this purpose, we substitute (18), (28) and (29) in (34) with due account for formula (30), as a result we obtain

$$
\widetilde{k}=\frac{E_{\infty}}{1-v_{\infty}^{2}}\left[1-\sum_{j=1}^{2} m_{j} \ni_{\gamma}^{*}\left(t_{j}^{\gamma}\right)\right]
$$

where

$$
m_{1}=\frac{3}{2} \frac{B\left(1-v_{\infty}\right)}{\left(1-2 v_{\infty}\right)}, \quad m_{2}=\frac{1}{2} \frac{D\left(1+v_{\infty}\right)}{\left(1-2 v_{\infty}\right)} .
$$

Considering Eqs. (35) and (21), the contact force is defined as

$$
P(t)=k_{\infty}\left[\alpha^{3 / 2}(t)-\sum_{j=1}^{2} m_{j} \int_{0}^{t} \ni_{\gamma}\left(-\frac{t-t^{\prime \prime}}{t_{j}}\right) \alpha^{3 / 2}\left(t^{\prime \prime}\right) d t^{\prime \prime}\right],
$$

where

$$
k_{\infty}=\frac{4 \sqrt{R}}{3} \frac{E_{\infty}}{1-v_{\infty}^{2}} .
$$

Now integrating Eq. (13) yields

$$
z=-\frac{1}{m} \int_{0}^{t} P\left(t^{\prime}\right)\left(t-t^{\prime}\right) d t^{\prime}+V_{0} t .
$$

Utilizing (36), it is possible to rewrite (37) in the form

$$
\begin{aligned}
& z(t)=V_{0} t-\frac{k_{\infty}}{m} \int_{0}^{t}\left[\alpha^{3 / 2}\left(t^{\prime}\right)\right. \\
& \left.-\sum_{j=1}^{2} m_{j} \int_{0}^{t^{\prime}} \ni_{\gamma}\left(-\frac{t^{\prime}-t^{\prime \prime}}{t_{j}}\right) \alpha^{3 / 2}\left(t^{\prime \prime}\right) d t^{\prime \prime}\right]\left(t-t^{\prime}\right) d t^{\prime} .
\end{aligned}
$$




\section{Solution of governing equations}

Now considering formulas (10) and (16), as well as relationship

$$
\frac{\partial w}{\partial z}=-G_{2}^{-1} W
$$

which is obtained from (3) if we substitute there the function $Z$ by the function $w$, Eq. (12) could be rewritten in the form

$$
M \alpha \dot{W}+g \alpha^{1 / 2} W=P(t),
$$

where contact force $P(t)$ is defined by (36), $M=\rho \pi h R$, and $g=2 M G_{2} R^{-1 / 2}$.

Substituting (11) in (13) yields

$$
m \dot{W}+m \ddot{\alpha}=-P(t) .
$$

Note that since the impact process is of short duration, then in the integrals entering in Eqs. (36) and (38) could be represented as [10]

$$
\ni_{\gamma}\left(-\frac{t}{t_{j}}\right) \approx \frac{t^{\gamma-1}}{t_{j}^{\gamma} \Gamma(\gamma)} \quad(j=1,2)
$$

The set of governing equations (40) and (41) with due account for (42) is reduced to the following:

$$
\begin{gathered}
M \alpha \dot{W}+g \alpha^{1 / 2} W=k_{\infty}\left[\alpha^{3 / 2}-\Delta_{\gamma} \int_{0}^{t}\left(t-t^{\prime}\right)^{\gamma-1} \alpha^{3 / 2}\left(t^{\prime}\right) d t^{\prime}\right], \\
m \dot{W}+m \ddot{\alpha}=-k_{\infty}\left[\alpha^{3 / 2}-\Delta_{\gamma} \int_{0}^{t}\left(t-t^{\prime}\right)^{\gamma-1} \alpha^{3 / 2}\left(t^{\prime}\right) d t^{\prime}\right]
\end{gathered}
$$

where

$$
\Delta_{\gamma}=\frac{1}{\Gamma(\gamma)}\left(\frac{m_{1}}{t_{1}^{\gamma}}+\frac{m_{2}}{t_{2}^{\gamma}}\right)
$$

\subsection{Analysis of the system's critical state}

The most interesting is the case of $N \rightarrow N_{\text {crit }}=K \mu h$, i.e., $G_{2} \rightarrow 0$, and the plate occurs in the critical state, since all energy of shock interaction is concentrated in the contact region, what may result in damage of the structure within the contact zone.

Let the compression force $N$ in the plate attain its critical magnitude. Then as a result of impact of a rigid body upon the plate, only one wave is generated in the plate which further propagates with the velocity $G_{1}$, but the second wave turns out to be locked within the contact region. In this case, coefficient $g=0$ and Eq. (43) is reduced to

$$
M \alpha \dot{W}=k_{\infty}\left[\alpha^{3 / 2}-\Delta_{\gamma} \int_{0}^{t}\left(t-t^{\prime}\right)^{\gamma-1} \alpha^{3 / 2}\left(t^{\prime}\right) d t^{\prime}\right] .
$$

If we consider

$$
\alpha \approx V_{0} t
$$

as a first approximation, then Eq. (45) with due account for [11]

$$
\int_{0}^{t}\left(t-t^{\prime}\right)^{\gamma-1} t^{\prime 3 / 2}\left(t^{\prime}\right) d t^{\prime}=\frac{3}{\gamma}\left(\frac{1}{3}-\frac{1}{5} \gamma\right) t^{3 / 2+\gamma}
$$

takes the form

$$
\dot{W}=\frac{k_{\infty} V_{0}^{1 / 2}}{M}\left[t^{1 / 2}-\Delta_{\gamma} \frac{3}{\gamma}\left(\frac{1}{3}-\frac{1}{5} \gamma\right) t^{1 / 2+\gamma}\right] .
$$

Integrating (48) yields

$$
W=\frac{k_{\infty} V_{0}^{1 / 2}}{M}\left[\frac{2}{3} t^{3 / 2}-\Delta_{\gamma} \frac{3}{\gamma}\left(\frac{1}{3}-\frac{1}{5} \gamma\right) \frac{1}{3 / 2+\gamma} t^{3 / 2+\gamma}\right] \text {. }
$$

\subsubsection{The case $\gamma=0$}

In the pure elastic case, i.e. at $\gamma=0, \Delta_{0}=\left.\Delta_{\gamma}\right|_{\gamma=0}=0$, and thus Eq. (45) takes the form

$$
\dot{W}=\frac{k_{\infty}}{M} \alpha^{1 / 2} .
$$
form

The solution of (50) with due account for (46) has the

$$
W=\frac{2 k_{\infty} V_{0}^{1 / 2}}{3 M} t^{3 / 2}
$$

whence it follows that the velocity of deflection increases as time goes on.

\subsubsection{The case $\gamma=1$}

In the case of conventional viscosity, i.e. at $\gamma=1$, Eq. (45) takes the form

$$
\dot{W}=\frac{k_{\infty}}{M}\left[\alpha^{1 / 2}-\frac{2}{5} \Delta_{1} \alpha^{3 / 2}\right],
$$

the solution of which is

$$
W=\frac{2 k_{\infty} V_{0}^{1 / 2}}{M}\left[\frac{1}{3}-\frac{2}{25} \Delta_{1} V_{0} t\right] t^{3 / 2},
$$

where

$$
\Delta_{1}=\left.\Delta_{\gamma}\right|_{\gamma=1}=\frac{m_{1}}{t_{1}}+\frac{m_{2}}{t_{2}} .
$$

Vanishing relationships (52) and (53) to zero, we could estimate the time at which velocity $W$ attains its maximal value and the contact duration, respectively,

$$
\begin{gathered}
t_{\max }=\frac{5}{2 \Delta_{1} V_{0}}, \\
t_{\text {cont }}=\frac{25}{6 \Delta_{1} V_{0}}=\frac{5}{3} t_{\max } .
\end{gathered}
$$

Substituting (54) in (53) provides the maximal magnitude of the velocity

$$
\left.W_{\max }\right|_{t=t_{\max }}=\frac{4}{15} \frac{k_{\infty} V_{0}^{1 / 2}}{M} t_{\max }^{3 / 2}
$$

whence it follows that viscosity softens the impact response of the plate as compared with the elastic case. 


\section{Conclusion}

The low-velocity impact response of a precompressed circular isotropic elastic plate is investigated. The dynamic behavior of the plate is described by equations taking the rotary inertia and transverse shear deformations into account. Longitudinal compressing forces are uniformly distributed along the plates median plane.

Contact interaction between the rigid impactor and the target is modeled by a generalized Hertz contact force, since it is assumed that the viscoelastic features of the plate represent themselves only in the place of contact and are governed by the standard linear solid model with fractional derivatives. This is explained by the fact that during the impact process, decrosslinking occurs within the domain of the contact of the plate with the sphere, resulting in more free displacements of molecules with respect to each other, and finally in the decrease of the plate material viscosity in the contact zone. This circumstance allows one to describe the behaviour of the plate material within the contact domain by the standard linear solid model involving fractional derivatives, since variation in the fractional parameter (the order of the fractional derivative) enables one to control the viscosity of the plate material.

From the results obtained the following conclusions may be drawn:

1. If a circular plate is subjected to the action of a constant compression force uniformly distributed in its middle plane along the boundary circumference, then during impact upon such a pre-stressed plate the nonstationary wave of a transverse shear (surface of strong discontinuity) is generated and then propagates with the velocity dependent on the compression force.

2. At certain critical magnitude of the compression force, the velocity of the transient wave of transverse shear vanishes to zero, resulting in 'locking' of this wave within the contact domain.

3. 'Locking' of the wave, in its turn, leads to the fact that energy during impact does not dissipate (as it takes place in the case of the generation and propagation of the transverse shear wave) but remains inside the contact zone, what could result in damage of the contact domain.

4. It is shown that for an elastic plate the critical compressional force leads to the increase in the velocity of the contact spot with time, resulting in cut off of the rigid washer (the contact zone) with further its knocking out of the plate.
5. If viscosity of the plate material is induced within the contact domain, then it softens the impact, and in this case the velocity of the contact spot continuously increases from zero to a certain maximal magnitude and then decreases to zero.

6. At fractional order viscosity, the maximal velocity of the contact spot could be controlled by the choice of the value of the fractional parameter.

\section{Acknowledgement}

The research described in this publication has been supported by the Ministry of Education and Science of the Russian Federation.

\section{References}

[1] S. Abrate, Impact on laminated composite materials, 2nd edition (Cambridge University Press, 2005)

[2] Yu.A. Rossikhin, M.V. Shitikova, The Shock and Vibration Digest 39, 273-309 (2007)

[3] A.C.J. Luo, Y. Guo, Vibro-impact dynamics (Wiley, 2013)

[4] R.A. Ibrahim, Vibro-impact dynamics: Modeling, mapping and applications (Springer, 2009)

[5] Yu.A. Rossikhin, M.V. Shitikova, Shock Vibr. 13, 197-214 (2006)

[6] Yu.A. Rossikhin, M.V. Shitikova, J. Sound Vibr. 330, 1985-2003 (2011)

[7] Yu.A. Rossikhin, M.V. Shitikova, Acta Mech. 102, 103-121 (1994)

[8] Yu.A. Rossikhin, M.V. Shitikova, Comp. Math. Appl. 66, 755-773 (2013)

[9] Yu.N. Rabotnov, Prikladnaya Matematika i Mekhanika (in Russian) 12, 53-62 (1948) (English translation of this paper could be found in Fract. Calc. Appl. Anal. 17, 684-696 (2014), doi:10.2478/s13540014-0193-1)

[10] Yu.A. Rossikhin, M.V. Shitikova, M.G. Estrada Meza, SpringerPlus 5:206 (2016), DOI 10.1186/s40064-016-1751-2

[11] Yu.A. Rossikhin, M.V. Shitikova, D.T. Manh, WSEAS Trans. Appl. Theor. Mech. 66, 125-128 (2016) 